\title{
Signes + et - : perception du circuit électrique
}

\section{A. Benseghir}

\section{(2) OpenEdition}

Journals

Édition électronique

URL : http://journals.openedition.org/trema/2311

DOI : 10.4000/trema.2311

ISSN : 2107-0997

\section{Éditeur}

Faculté d'Éducation de l'université de Montpellier

\section{Édition imprimée}

Date de publication : 1 mai 1993

Pagination : 19-25

ISSN : 1167-315X

\section{Référence électronique}

A. Benseghir, «Signes + et - : perception du circuit électrique », Tréma [En ligne], 3-4 | 1993, mis en ligne le 01 mai 1993, consulté le 30 avril 2019. URL : http://journals.openedition.org/trema/2311 ; DOI :

10.4000/trema.2311

Ce document a été généré automatiquement le 30 avril 2019

Trema 


\section{Signes + et - : perception du circuit électrique}

\section{A. Benseghir}

1 Le langage de la physique met en jeu une diversité de représentations symboliques pour traduire certains aspects du réel. Dans l'enseignement, celles-ci peuvent induire chez l'élève des effets perceptifs et conceptuels inattendus (Fischbein, 1969 ; Johsua, 1982). Une sémantique "personnelle» des représentations en cause peut ainsi s'élaborer au détriment de la sémantique correspondante admise.

2 Les signes + et -, utilisés en électricité semblent potentiellement illustrer l'hypothèse ci dessus. Sur le plan didactique, le rapport entre ces signes et l'idée d'accumulation de charges est fréquemment mis en exergue dans les contenus d'enseignement de l'électrostatique (Benseghir, 1989a). En électrocinétique, les signes + et -, associés aux pôles du générateur, sont évoqués en général lors de l'introduction de ce dernier et à l'occasion de la définition du sens conventionnel du courant électrique. Dans les manuels français et algériens du secondaire de la dernière décennie, la sémantique de ces signes semble relever, au moins partiellement, de l'implicite.

3 Le caractère arbitraire et la référence aux potentiels le plus élevé et le moins élevé des mêmes signes, n'y sont souvent évoqués que de manière allusive. Tenant compte de ces données, cet exposé tentera d'éclairer les difficultés liées au changement de sémantique des signes + et - correspondant à la transition électrostatique-électrocinétique. Dans ce sens on présentera d'abord, pour mieux préciser la problématique ci-dessus, un aperçu historique relatif à l'émergence de ces signes en électricité. Des résultats d'une enquête permettront ensuite d'étayer l'hypothèse directrice de cette étude. 


\section{Emergence des signes + et - dans le champ sémantique de l'électricité}

\subsection{L'étape électrostatique}

Jusqu'à la fin du XVIIIème, la science de l'électricité se confine encore dans l'étude des phénomènes d'électricité statique, les attractions et les répulsions entre corps chargés d'une part, et les décharges d'autre part. La mise en évidence empirique de deux espèces d'électricité (Dufay, 1733) fait naître tôt le besoin de dénommer celles-ci. Deux théories concurrentes de l'électricité dominent à l'époque. La théorie du fluide unique, énoncée par Franklin en 1750, considère qu'un corps neutre contient une certaine quantité de substance spécifique, le fluide électrique. L'excès ou le défaut de ce fluide par rapport à cette "quantité normale» rend compte de l'une ou de l'autre des deux espèces d'électricité dont un corps peut se trouver affecté. L'autre théorie, défendue surtout en France (Symmer, 1759) admet l'existence de deux fluides électriques, le vitré et le résineux, la neutralité d'un corps étant interprétée par l'absence de ceux-ci dans le corps considéré. Inspiré probablement par la connotation algébrique d'une autre donnée empirique, la possibilité de neutralisation réciproque des deux espèces d'électricité, le choix des signes + et - et des termes positif et négatif pour désigner celles-ci, est adopté dans les deux théories.

\subsection{Transition électrostatique-électrocinétique}

5 L'étude du circuit électrique (fermé) n'est amorcée qu'à partir de 1789 avec les expériences de Galvani, dans une ambiance de familiarisation avec l'électricité statique. La mise au point en 1800 de l'Appareil électro-moteur par Volta met fin à une période de doute concernant la nature électrique des phénomènes du cercle galvanique. La pile de Volta, appréhendée alors au travers du système conceptuel et instrumental dominant électrostatique en l'occurrence-, est, du fait de l'électrisation de ses extrémités, comparée à une bouteille de Leyde comme en témoigne ce passage: «...et la pile, nous ne nous lassons point de le répéter, doit être considérée comme une bouteille, dans sa manière d'accumuler et de retenir le fluide » (Gérard et Vaw Mons, 1802).

6 Aux pôles de la pile, considérés comme sources des fluides vitrés et résineux, est souvent attribuée la causalité des effets chimiques, magnétiques, etc., au même titre que la causalité des effets électroscopiques (attractions, répulsions) (Cuvier, 1801). C'est dans cette optique électrostatique que les signes + et - semblent être associés à ces pôles (De la Rive, 1833).

7 A partir de 1820, avec les expériences d'Oersted, la causalité polaire fait place, non sans difficultés, à la notion de circuit électrique dans l'aspect opératoire essentiellement (Benseghir, 1989b). L'idée de courant qui émerge alors reste encore très empreinte de celle de décharge. C'est la conception du double courant (Ampère, 1820) que Lamé (1837) explicite ainsi: "On conçoit que dans une pile dont les extrémités sont en communication, l'équilibre électrique est impossible car d'une part [...] les actions chimiques qui s'y passent, tendent toujours à accumuler vers les extrémités des fluides contraires et d'autre part, le conducteur interpolaire réunit incessamment les électricités accumulées ». 


\subsection{Définition empiriste du courant électrique}

Dans les expériences ayant suivi celles d'Oersted, les physiciens constatent que l'aiguille aimantée dévie à proximité de toutes les parties du circuit électrique fermé. Boisgiraud par exemple, manipulant deux piles reliées en série, remarque avec surprise: "Cette disposition d'appareil me fournit l'occasion de m'assurer que l'arc métallique, qui joignait les pôles extrêmes, n'était pas le seul susceptible de faire dévier l'aiguille; l'arc intermédiaire, qui réunissait l'une des piles à l'autre, produisait le même effet et suivant les mêmes lois » (1820). L'idée de circulation complète du courant, suggérée par de tels faits expérimentaux (Ampère, 1820 ; De la Rive, 1825), s'accorde mal avec le modèle du double courant. Pour surmonter cette difficulté, Ampère élabore une définition empiriste du sens du courant électrique: "L'expression: direction du courant dans la pile, désignera la direction qui va de l'extrémité où l'hydrogène se dégage dans la décomposition de l'eau, à celle où l'on obtient de l'oxygène »; et celle-ci : «Direction du courant électrique dans le conducteur qui établit la communication entre les deux extrémités de la pile, désignera la direction qui va, au contraire, de l'extrémité où se produit l'oxygène à celle où se développe l'hydrogène ». «Au reste, tout ce que je dis sur ce sujet ne suppose aucunement qu'il y ait réellement un courant dans cette direction, et on ne peut considérer que comme une manière commode et usitée de la désigner, l'emploi que je fais de cette dénomination de courant électrique » (1820). Dépouillés du caractère électrostatique qui leur était habituellement affecté, les pôles de la pile sont réduits dans cette définition au statut de simples «sortie » et « entrée » du courant. En fait, il faut attendre la consécration de la théorie d'Ohm (à «fluide unique ») vers 1850 pour que cette manière de traiter le circuit électrique ait raison du modèle du double courant.

9 Le contexte historique d'appréhension des phénomènes de courant présentant une certaine analogie avec le contexte actuel d'apprentissage de l'électrocinétique, les données dégagées ci-dessus semblent conforter épistémologiquement l'idée d'une « contamination » électrostatique de la sémantique des étudiants à propos des signes + et - en électrocinétique.

\section{Signes + et - en électrocinétique : sémantique des étudiants}

Les résultats suivants sont extraits d'une étude empirique relative à la transition électrostatique-électrocinétique axée sur l'hypothèse d'une influence des connaissances électrostatiques des étudiants sur leur approche du circuit électrique. Des échantillons d'élèves du second cycle du secondaire, algériens (2èmeAS -année secondaire-, 3èmeAS) et français (2nde, 1ère) et d'étudiants de début d'université (Univ.l, Algérie; DEUG1, DEUG2, France) ont été interrogés après enseignement. Les questions ont trait à l'existence d'une d.d.p. et indirectement à celle d'un courant électrique entre deux points appartenant à des circuits de configurations variées. 


\subsection{Perception des pôles + et -}

11 Au moyen de la situation 1 (fig.1) on voulait préciser le rôle imparti par les étudiants aux signes + et - en circuit fermé. A la question sur la possibilité d'allumage de l'ampoule, la majorité des étudiants interrogés répondent par la négative (tableau I).

Situation 1

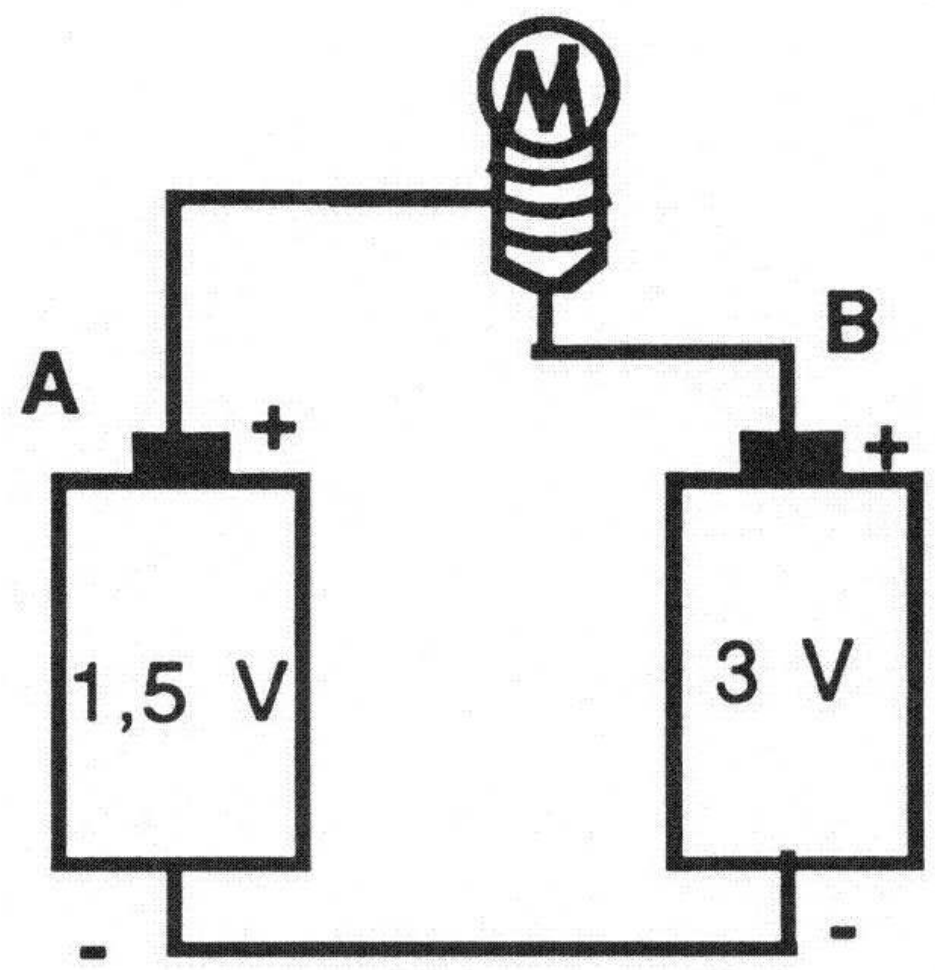

Figure 1 


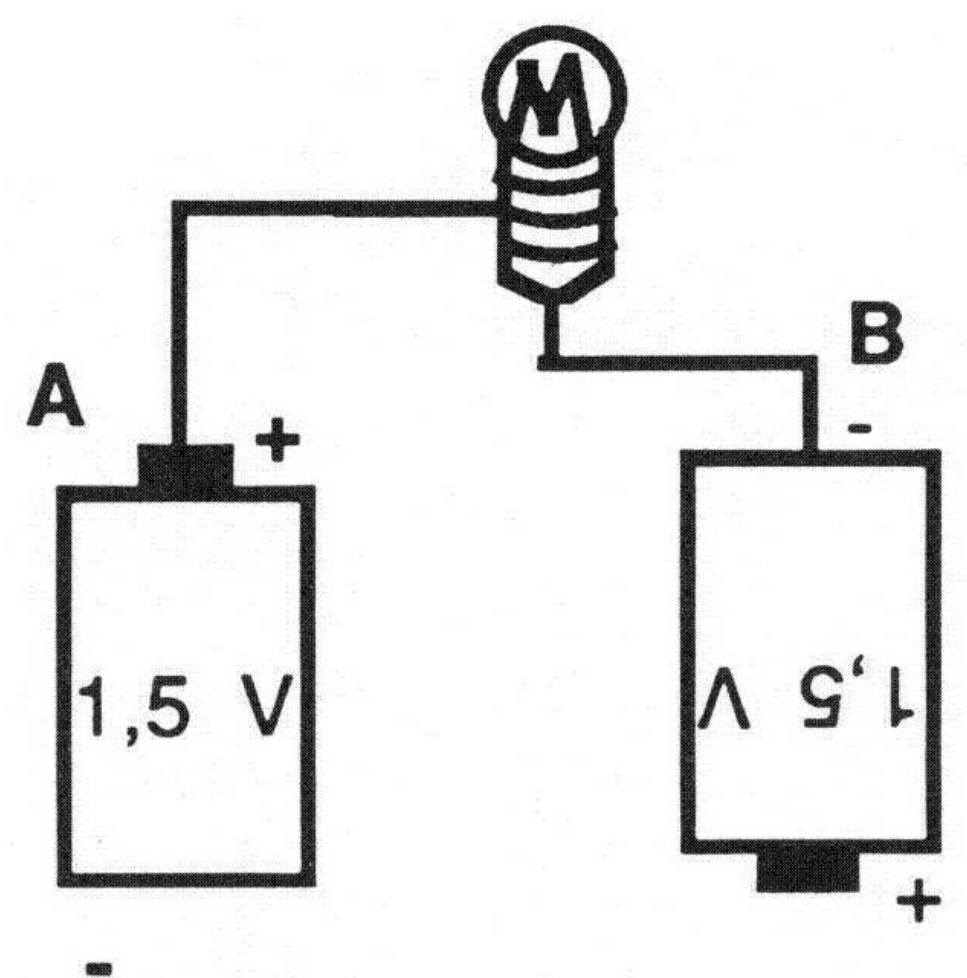

Figure 2

Tableau I : situation 1, fréquences des réponses NON (incorrectes)

\begin{tabular}{|c|c|c|}
\hline $\begin{array}{c}\text { 1ère (Fr.) } \\
(103)^{* *}\end{array}$ & $\begin{array}{c}\text { 3ème AS (Alg.) } \\
(71)^{* *}\end{array}$ & $\begin{array}{c}\text { Univ. 1 (Alg.) } \\
(45) * *\end{array}$ \\
\hline $90 \%$ & $77 \%$ & $55 \%$ \\
\hline
\end{tabular}

**Effectif de l'échantillon

12 L'argument principal présenté dans les commentaires des réponses est la similitude de signe des charges aux pôles A et B comme le montre cet exemple ( $\left.1^{\text {ére }}\right)$.

Cette focalisation sur les pôles, perçus comme chargés, et dont le signe semble indiquer la nature de la charge, est confirmé au moyen de la situation 2 (fig.2). Prés du 1/5 des échantillons français et de la moitié des échantillons algériens considèrent l'ampoule comme allumée ! (tableau II).

Tableau II : situation 2, fréquences des réponses OUI (incorrectes).

\begin{tabular}{|c|c|c|c|}
\hline $\begin{array}{c}\text { 2nde } \\
(53)\end{array}$ & $\begin{array}{c}\text { DEUG 1 } \\
(83)\end{array}$ & $\begin{array}{c}\text { 2nde AS } \\
(112)\end{array}$ & $\begin{array}{c}\text { Univ.1 } \\
(94)\end{array}$ \\
\hline $19 \%$ & $16 \%$ & $55 \%$ & $51 \%$ \\
\hline
\end{tabular}


14 Les justifications correspondantes ressemblent, a contrario, aux précédentes (situation 1) : «[...] puisqu'il existe une différence de charges entre les points A et B donc il y aura sûrement passage d'électrons de B à A » (Univ.1, Alg.).

\subsection{Sens conventionnel et approche du circuit électrique}

Pour une proportion importante d'étudiants les signes + et - associés aux pôles semblent donc renvoyer à l'idée d'accumulation de charge. Les parties du circuit concernées (ici les pôles) constituent en fait les points d'ancrage d'une approche particulière de celui-ci. La définition du sens conventionnel, élément fourni par l'enseignement, n'apparaît dans les justifications des étudiants que comme un schème rigidifié, plaqué sur une interprétation profondément électrostatique du circuit électrique : «Car le courant va du plus vers le moins donc une pile va se décharger pendant que l'autre va se charger. " (1ère, réponse oui, situation 2). «Le courant va toujours du + vers le - et ici les électrons ne sont pas attirés par B. Le courant ne passe pas » (situation 1, réponse NON, 1ère.).

\subsection{Points d'ancrage de l'approche électrostatique}

D'autres situations où les pôles n'interviennent pas directement dans les questions posées ont été testées, parmi lesquelles les situations 3 et 4 (fig.3 et 4).

Situation 3 (figure 3)

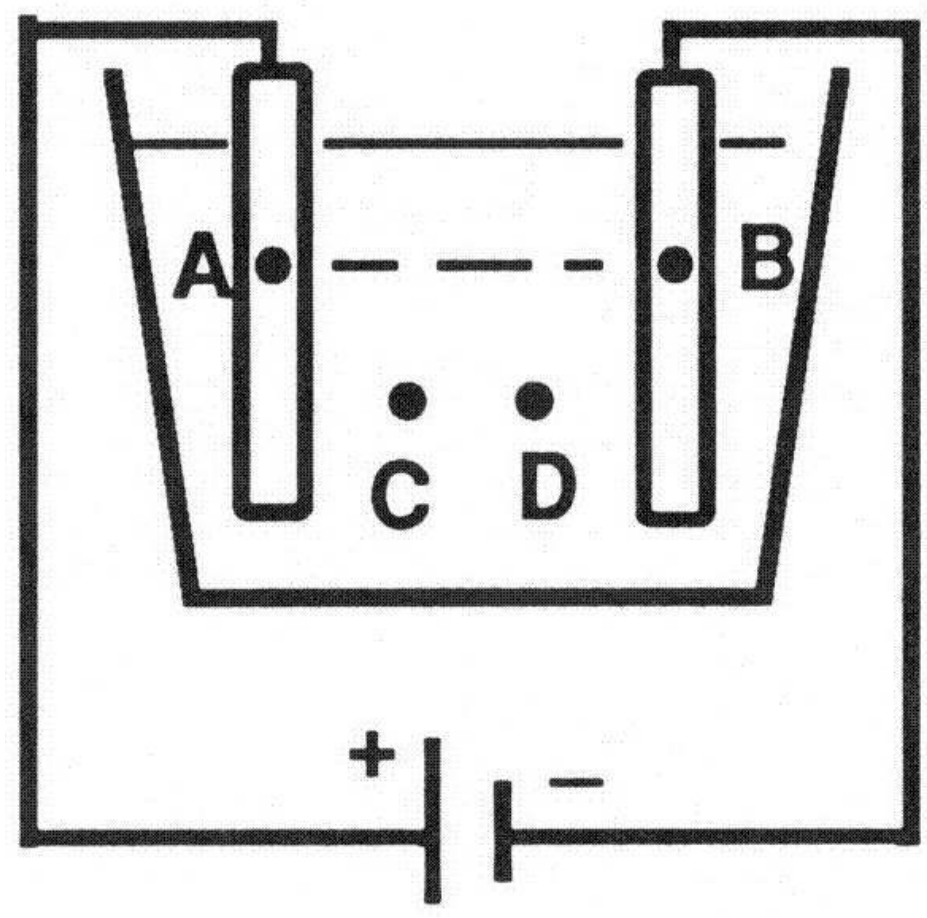




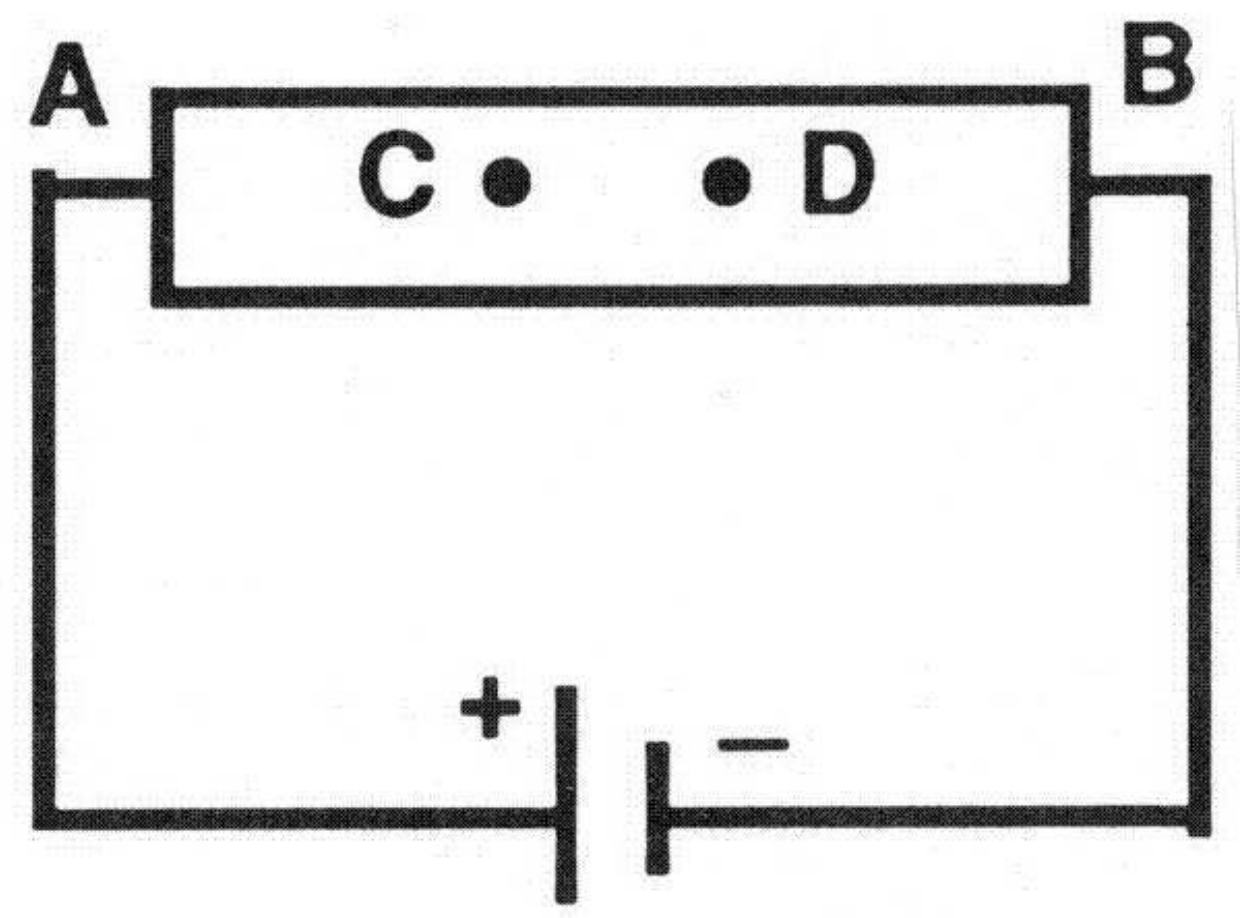

17 La question concerne l'existence d'une d.d.p. entre les points $C$ et $D$, internes à la résistance ou à la solution (électrolyseur).

Plus du $1 / 3$ des étudiants universitaires et la majorité des élèves de 1ère interrogés, choisissent à propos de la situation 3 (les résultats obtenus avec cette situation sont similaires à ceux relatifs à la situation 4), la réponse incorrecte NON (tableau III). Dans les commentaires correspondants, les points $C$ et $D$ sont considérés comme des points non singuliers, c'est-à-dire quelconques, contrairement aux pôles du générateur ou des bornes des récepteurs auxquels il est plus ou moins explicitement associé une dissymétrie de signes des charges : «Les points $C$ et $D$ ne sont reliés à aucun générateur et malgré qu'ils soient dans une solution conductrice, ils ne présentent pas une différence de polarité » (2nde). "Car, contrairement aux points $A$ et $B$ où l'on trouve d'une part une accumulation de charges et d'autre part un déficit de charges, entre les points $C$ et $D$, les électrons circulent de façon homogène et se trouvent assez dilués » (DEUG.2).

Tableau III : Situation 3 : Fréquences des réponses NON.

\begin{tabular}{|c|c|c|}
\hline $\begin{array}{c}\text { lère } \\
(100)\end{array}$ & $\begin{array}{c}\text { DEUG 2 } \\
(32)\end{array}$ & $\begin{array}{c}\text { Univ. 1 } \\
(24)\end{array}$ \\
\hline $81 \%$ & $38 \%$ & $35 \%$ \\
\hline
\end{tabular}




\section{Conclusion}

Les signes + et - constituent un exemple de représentations symboliques caractérisées par un dédoublement sémantique lié à une utilisation commune dans des domaines distincts du savoir. Cet aspect, souvent occulté dans l'enseignement, peut être source de difficultés didactiques insoupçonnées.

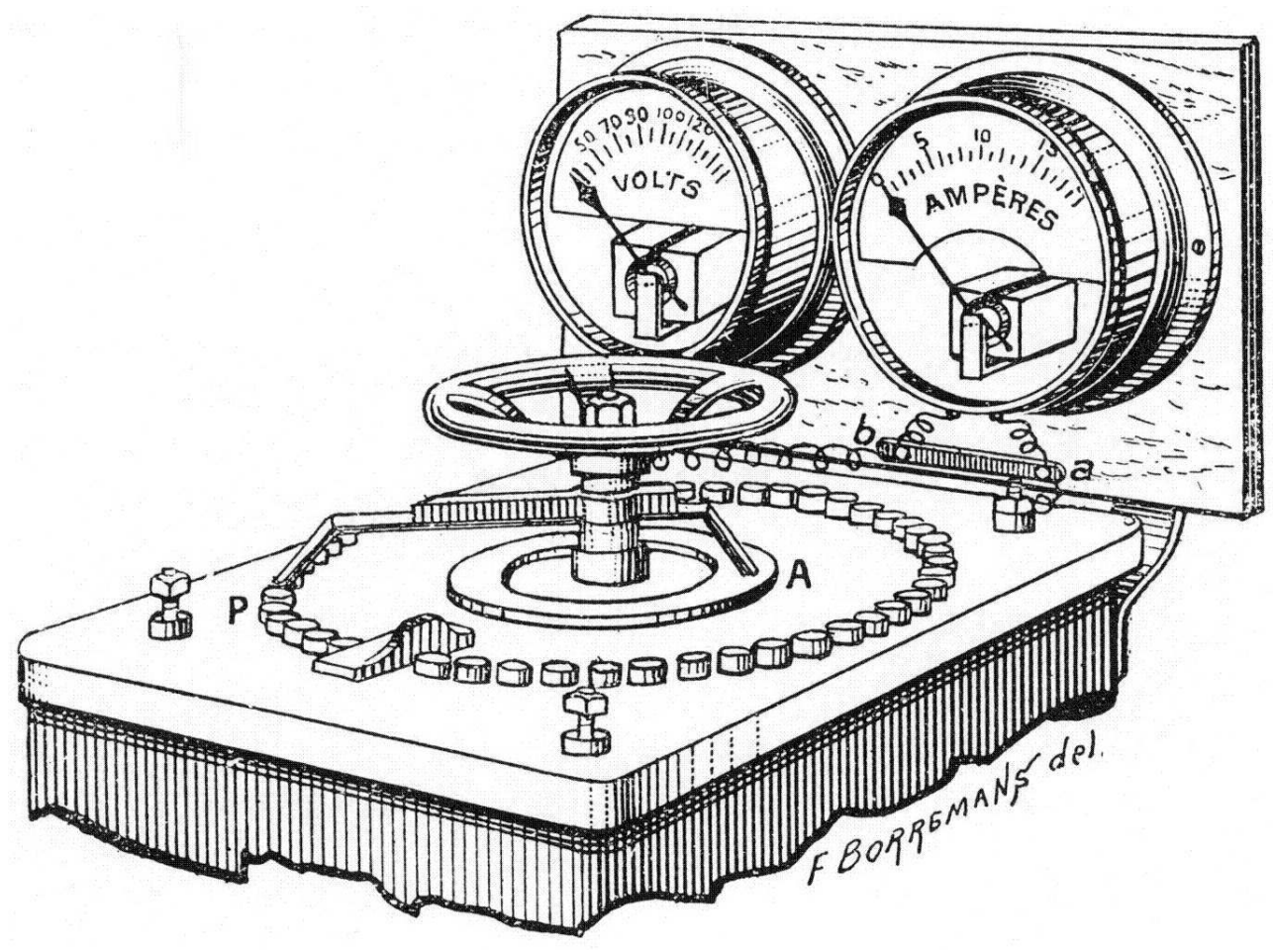

\section{BIBLIOGRAPHIE}

\section{Références}

AMPERE, A.M. De l'action exercée sur un courant par un autre, le globe terrestre ou un aimant. Annales de physique et de chimie, 15, 59-76, 170-218, 1820.

BENSEGHIR, A., a) Transition électrostatique-électrocinétique : point de vue historique et analyse des difficultés des élèves. Thèse nouveau régime, Université Paris VII, 1989.

BENSEGHIR, A., b) Formation des concepts d'électrocinétique : point de vue historique. Bulletin de l'union des physiciens, avril 1989.

BOISGIRAUD ainé. De l'action de la pile sur l'aiguille aimantée. Annales de chimie et de physique, 15, 279-289, 1820.

CUVIER : Rapport sur le galvanisme. Journal de physique, 52, 318-324, 1820. 
DE LA RIVE, A. : Mémoire sur quelques-uns des phénomènes que présente l'électricité dans son passage à travers les conducteurs liquides. A.C.P., 28, 190-221, 1825.

DE LA RIVE, A. : Esquisse historique des principales découvertes faites dans l'électricité depuis quelques années. Bibliothèque universelle, sciences et arts, 52, 225-264, 1833.

FISCHBEIN, E. : Modèles figuraux implicites et enseignement scientifique. Revue roumaine des sciences sociales, 14, 79-85., 1969.

GERARD, F. et VAN MONS, J.B. : Sur les expériences confirmant l'identité du fluide électrique avec le fluide qui fait naître les phénomènes galvaniques. Journal de chimie, 1, 191-213, 1802.

LAME, G. : Cours de l'Ecole polytechnique, tome 2, 2ème partie, 1837.

JOHSUA, S. : L'utilisation du schéma en électrocinétique : aspects perceptifs et aspects conceptuels. Thèse de 3ème cycle. Université de Provence, 1982.

OERSTED, J.C. : Expérience sur l'effet du conflit électrique sur l'aiguille aimantée. A.C.P, 14, $417-425,1820$

\section{RÉSUMÉS}

Les représentations symboliques utilisées en physique (schémas, signes, etc.) font rarement l'objet d'un enseignement explicite. Il est plausible que les étudiants suppléent à cette carence en formant leur propre sémantique. Ainsi les signes + et - employés en électrocinétique semblent renvoyer pour une proportion significative d'étudiants à la désignation électrostatique. Ce comportement va de pair avec une analyse du circuit électrique privilégiant certains points de celui-ci, notamment les pôles de générateurs, perçus comme chargés.

Symbolic representations used in physics (Schemas, signs, etc.), are rarely explicitly taught. It is plausible that students supply this failure, by elaborating their own semantic. Thus, for significant number of students the + and - signs employed in electro kinetics, seem to have the same indication as in electrostatics. This behaviour keeps up with a specific approach of electric circuit, which privileges certain points, especially generator poles, perceived as charged.

INDEX

Mots-clés : électrocinétique, électrostatique, histoire, représentation symbolique, signe

\section{AUTEUR}

\section{A. BENSEGHIR}

Institut de Physique, Université de Sétif, Algérie et LDPES, Université Paris VII 